\title{
Experimentelle Studien über die Funktion des reticuloendothelialen Systems
}

\author{
IV. Mitteilung \\ Bedeutung der Milz im Reticuloendothelialen System \\ Von \\ Shoichi Yamagata, Toshio Aratani, Kiyomi Miura und \\ (山形 敉 一) \\ （管谷利夫）（三浦清美） \\ Kisao Otomo \\ (大友喜侁雄) \\ Aus der Med. Klinik von Prof. Dr. T. Kurokawa, Tohoku \\ Universität, Sendai
}

(Received for publication, February 2, 1954)

Seit Aschoff ${ }^{11}$ ist es allgemein anerkannt, dass die Milz ein wichtiges Organ des reticuloendothelialen Zellapparats ist. Aber Akazaki ${ }^{2}$ ' betont, dass die Milzsinusendothelien zwischen den eigentlichen Endothelien und Reticuloendothelien stehen, weil sie sowohl funktionell als auch morphologisch als Reticuloendothelien nur dann wirken können, wenn sie besonders stark stimuliert werden.

Nach unserer früheren Mitteilung ${ }^{3)}$ kann der Kongorotindex die Funktion des RES und die Prothrombinzahl die Leberzellenfunktion verraten; deshalb wurde in der vorliegenden Untersuchung untersucht, wie die Milz auf die Funktion des RES und der Leber einwirkt. Die Auswahl der Versuchstiere sowie die Methodik war die gleiche wie bei der I. Mitteilung ${ }^{4}$, und wir verglichen den Durchschnittswert von 2 oder 3 Kaninchen mit dem bei den Belastungsversuchen in der I. Mitteilung.")

\section{Versuchsergebnisse}

1. Einfluss der Milzexstirpation

Wie aus Tabelle I und II zu ersehen, bleiben der Kongorotindex und die Prothrombinzahl bei Laparotomieversuchen gegenüber den Kontrollversuchen fast unverändert, mit geringer Ausnahme des Kongorotindex für eine kurze Zeitspanne nach Laparotomieversuchen. Aber der Kongorotindex vermehrt sich bei der Milzexstirpation bis etwa zum 7 . Tage, dann vermindert er sich und kehrt ungefähr nach 4 Wochen zum früheren Zustand wieder zurück. In diesem Falle ist eine Verminderung der Prothrombinzahl nicht so auffallend.

Wenn man 2 Leberlappen des Kaninchens am 18. Tage nach der 
TABELLE I

Einfluss der Milzexstirpation für eine kurze Zeitspanne

\begin{tabular}{|c|c|c|c|c|c|c|c|c|c|}
\hline & \multirow[t]{2}{*}{ " } & \multirow{2}{*}{$\begin{array}{l}\text { Nr. des } \\
\text { Kaninchens }\end{array}$} & \multirow{2}{*}{$\begin{array}{l}\text { Vor. } \\
\text { Op. }\end{array}$} & \multicolumn{6}{|c|}{ Zeit nach Operation (St.) } \\
\hline & & & & 3 & 5 & 8 & 12 & 24 & 48 \\
\hline \multirow[t]{2}{*}{ Kontrolle } & K-index & $\begin{array}{c}1 \\
2 \\
3 \\
4 \\
\text { Durchschn. } \\
\text { Differenz }\end{array}$ & $\begin{array}{l}62.6 \\
54.5 \\
37.5 \\
35.1 \\
47.3\end{array}$ & $\begin{array}{r}66.3 \\
57.2 \\
49.9 \\
44.6 \\
54.1 \\
6.8\end{array}$ & $\begin{array}{l}66.7 \\
60.0 \\
56.6 \\
58.2 \\
60.8 \\
13.5\end{array}$ & $\begin{array}{l}69.4 \\
60.0 \\
50.0 \\
47.7 \\
58.1 \\
10.8\end{array}$ & $\begin{array}{r}64.5 \\
55.7 \\
40.7 \\
56.3 \\
54.8 \\
7.5\end{array}$ & $\begin{array}{r}58.8 \\
58.2 \\
44.3 \\
53.3 \\
54.1 \\
6.8\end{array}$ & \begin{tabular}{r|}
65.8 \\
54.0 \\
54.8 \\
46.1 \\
56.1 \\
8.8
\end{tabular} \\
\hline & P-zahl & \begin{tabular}{l}
1 \\
\multicolumn{1}{c}{1} \\
2 \\
3 \\
Durchschn. \\
Differenz
\end{tabular} & $\begin{array}{l}1.00 \\
1.00 \\
1.00 \\
1.00\end{array}$ & $\begin{array}{c}1.03 \\
1.01 \\
0.95 \\
1.00 \\
0\end{array}$ & $\begin{array}{c}1.03 \\
0.99 \\
0.98 \\
1.00 \\
0\end{array}$ & $\begin{array}{l}1.01 \\
1.01 \\
1.01 \\
1.01 \\
0.01\end{array}$ & $\begin{array}{r}0.98 \\
0.97 \\
1.01 \\
0.99 \\
-0.01\end{array}$ & $\begin{array}{c}1.01 \\
0.99 \\
1.01 \\
1.00 \\
0\end{array}$ & $\begin{array}{r}0.98 \\
0.96 \\
0.95 \\
0.96 \\
-0.04\end{array}$ \\
\hline \multirow{2}{*}{ Laparotomie } & $\mathrm{K}$-index & \begin{tabular}{l}
\multicolumn{1}{c}{1} \\
2 \\
3 \\
Durchschn. \\
Differenz
\end{tabular} & $\begin{array}{l}71.3 \\
50.0 \\
47.0 \\
55.8\end{array}$ & $\begin{array}{l}72.6 \\
53.5 \\
50.0 \\
57.6 \\
-5.0\end{array}$ & $\begin{array}{l}72.8 \\
65.0 \\
58.2 \\
65.8 \\
-3.5\end{array}$ & $\begin{array}{l}78.3 \\
53.7 \\
62.6 \\
62.7 \\
-3.0\end{array}$ & $\begin{array}{r}70.3 \\
64.4 \\
59.2 \\
65.0 \\
1.7\end{array}$ & $\begin{array}{r}62.2 \\
52.7 \\
44.2 \\
51.2 \\
-10.9\end{array}$ & $\begin{array}{r}60.0 \\
55.2 \\
44.2 \\
52.4 \\
-12.2\end{array}$ \\
\hline & P-zahl & \begin{tabular}{l}
1 \\
\multicolumn{1}{c}{1} \\
2 \\
3 \\
Durchschn. \\
Differenz
\end{tabular} & $\begin{array}{l}1.00 \\
1.00 \\
1.00 \\
1.00\end{array}$ & $\begin{array}{l}1.01 \\
1.02 \\
1.00 \\
1.01 \\
0.01\end{array}$ & $\begin{array}{c}0.99 \\
1.00 \\
1.00 \\
1.00 \\
0\end{array}$ & $\begin{array}{l}1.00 \\
1.02 \\
1.00 \\
1.01 \\
0\end{array}$ & $\begin{array}{l}1.01 \\
1.02 \\
0.99 \\
1.01 \\
0.02\end{array}$ & $\begin{array}{l}1.01 \\
1.01 \\
0.97 \\
1.00 \\
0\end{array}$ & $\begin{array}{l}1.03 \\
1.00 \\
0.95 \\
0.99 \\
0.03\end{array}$ \\
\hline \multirow{2}{*}{ Milzexstirpation } & K-index & \begin{tabular}{l}
\multicolumn{1}{c}{1} \\
2 \\
Durchschn. \\
Differenz
\end{tabular} & $\begin{array}{l}55.2 \\
55.0 \\
55.1\end{array}$ & $\begin{array}{r}61.8 \\
65.6 \\
64.1 \\
7.2\end{array}$ & $\begin{array}{l}84.1 \\
75.2 \\
78.1 \\
13.0\end{array}$ & $\begin{array}{l}82.0 \\
77.0 \\
78.8 \\
16.8\end{array}$ & $\begin{array}{r}82.7 \\
62.5 \\
67.1 \\
2.8\end{array}$ & $\begin{array}{r}51.7 \\
73.0 \\
59.1 \\
8.1\end{array}$ & $\begin{array}{l}57.8 \\
68.0 \\
65.0 \\
13.3\end{array}$ \\
\hline & P-zahl & $\begin{array}{l}\quad 1 \\
\quad 2 \\
\text { Durchschn. } \\
\text { Differenz }\end{array}$ & $\begin{array}{l}1.00 \\
1.00 \\
1.00\end{array}$ & $\begin{array}{r}1.00 \\
0.99 \\
1.00 \\
-0.01\end{array}$ & $\begin{array}{c}0.99 \\
1.00 \\
1.00 \\
0\end{array}$ & $\begin{array}{l}1.01 \\
1.03 \\
1.02 \\
0.02\end{array}$ & $\begin{array}{r}1.02 \\
1.00 \\
1.01 \\
-0.01\end{array}$ & $\begin{array}{l}1.04 \\
0.97 \\
1.01 \\
0.01\end{array}$ & $\begin{array}{r}1.05 \\
0.98 \\
1.02 \\
-0.01\end{array}$ \\
\hline \multirow{2}{*}{$\begin{array}{l}2 \text { Leberlappen- } \\
\text { resektionen } \\
\text { nach Milzex- } \\
\text { stirpation }\end{array}$} & $\mathrm{K}$-index & \begin{tabular}{l}
\multicolumn{1}{c}{1} \\
\multicolumn{2}{c}{} \\
Durchschn. \\
Differenz.
\end{tabular} & $\begin{array}{l}56.6 \\
49.5 \\
53.2\end{array}$ & $\begin{array}{l}70.1 \\
78.1 \\
73.8 \\
25.6\end{array}$ & $\begin{array}{l}72.7 \\
76.3 \\
74.2 \\
24.5\end{array}$ & $\begin{array}{l}76.0 \\
89.4 \\
82.6 \\
32.4\end{array}$ & $\begin{array}{l}78.8 \\
84.1 \\
81.4 \\
26.5\end{array}$ & $\begin{array}{l}64.2 \\
74.0 \\
69.7 \\
27.4\end{array}$ & $\begin{array}{l}56.3 \\
70.7 \\
63.4 \\
22.4\end{array}$ \\
\hline & P-zahl & \begin{tabular}{l}
$\quad 1$ \\
\multicolumn{1}{c}{1} \\
Durchschn. \\
Differenz
\end{tabular} & $\begin{array}{l}1.00 \\
1.00 \\
1.00\end{array}$ & $\begin{array}{r}0.94 \\
0.97 \\
0.96 \\
-0.05\end{array}$ & $\begin{array}{r}0.97 \\
0.90 \\
0.94 \\
-0.06\end{array}$ & $\begin{array}{r}0.93 \\
0.86 \\
0.90 \\
-0.11\end{array}$ & $\begin{array}{r}0.94 \\
0.85 \\
0.90 \\
-0.11\end{array}$ & $\begin{array}{r}0.90 \\
0.87 \\
0.89 \\
-0.12\end{array}$ & $\begin{array}{r}0.94 \\
0.87 \\
0.91 \\
-0.10\end{array}$ \\
\hline \multirow{2}{*}{$\begin{array}{l}2 \text { Leberlappen } \\
\text { resektionen }\end{array}$} & K-index & \begin{tabular}{l}
\multicolumn{1}{c}{1} \\
2 \\
Durchschn. \\
Differenz
\end{tabular} & $\begin{array}{l}57.8 \\
48.7 \\
52.8\end{array}$ & $\begin{array}{r}61.6 \\
77.1 \\
68.5 \\
8.7\end{array}$ & $\begin{array}{l}69.9 \\
77.7 \\
73.8 \\
12.5\end{array}$ & $\begin{array}{l}75.0 \\
80.2 \\
77.8 \\
18.5\end{array}$ & $\begin{array}{l}71.0 \\
66.7 \\
68.3 \\
13.2\end{array}$ & $\begin{array}{r}60.6 \\
60.6 \\
60.6 \\
5.1\end{array}$ & $\begin{array}{r}66.5 \\
57.3 \\
61.7 \\
6.5\end{array}$ \\
\hline & P-zahl & \begin{tabular}{l}
\multicolumn{1}{c}{1} \\
2 \\
Durchschn. \\
Differenz
\end{tabular} & $\begin{array}{l}1.00 \\
1.00 \\
1.00\end{array}$ & $\begin{array}{r}0.97 \\
0.81 \\
0.89 \\
-0.12\end{array}$ & $\begin{array}{r}0.99 \\
0.93 \\
0.96 \\
-0.04\end{array}$ & $\begin{array}{r}0.99 \\
0.93 \\
0.95 \\
-0.06\end{array}$ & $\begin{array}{r}0.98 \\
0.90 \\
0.94 \\
-0.07\end{array}$ & $\begin{array}{r}0.99 \\
0.90 \\
0.95 \\
-0.04\end{array}$ & $\begin{array}{r}1.03 \\
0.93 \\
0.98 \\
-0.02\end{array}$ \\
\hline
\end{tabular}


TABELLE II

Einfluss der Milzexstirpation im langen Intervalle

\begin{tabular}{|c|c|c|c|c|c|c|c|c|c|c|c|}
\hline & & \multirow{2}{*}{$\begin{array}{c}\text { Nr. des } \\
\text { Kaninchens }\end{array}$} & \multirow{2}{*}{$\begin{array}{l}\text { Vor } \\
\text { Op. }\end{array}$} & \multicolumn{8}{|c|}{ Zeit nach Operation (Tage) } \\
\hline & & & & 1 & 2 & 4 & 7 & 10 & 14 & 21 & 28 \\
\hline \multirow{2}{*}{ Kontrolle } & $\mathrm{K}$-index & \begin{tabular}{l}
\multicolumn{1}{c}{1} \\
\multicolumn{1}{c}{3} \\
3 \\
Durchschn. \\
Differenz
\end{tabular} & $\begin{array}{l}49.5 \\
45.6 \\
44.7 \\
47.6\end{array}$ & $\begin{array}{r}51.8 \\
47.3 \\
44.1 \\
47.8 \\
0.2\end{array}$ & $\begin{array}{l}45.0 \\
46.3 \\
41.1 \\
44.7 \\
-2.9\end{array}$ & $\begin{array}{l}44.0 \\
40.0 \\
41.9 \\
41.8 \\
-5.8\end{array}$ & $\begin{array}{l}49.8 \\
39.3 \\
46.8 \\
45.3 \\
-2.3\end{array}$ & $\begin{array}{l}39.8 \\
35.3 \\
51.5 \\
42.5 \\
-5.1\end{array}$ & $\begin{array}{l}51.0 \\
46.9 \\
42.3 \\
47.3 \\
-0.3\end{array}$ & $\begin{array}{r}53.8 \\
55.5 \\
43.3 \\
51.2 \\
3.6\end{array}$ & $\begin{array}{r}58.6 \\
45.8 \\
52.3 \\
4.7\end{array}$ \\
\hline & P-zahl & \begin{tabular}{l}
\multicolumn{1}{c}{1} \\
\multicolumn{2}{|c}{} \\
3 \\
Durchschn. \\
Differenz
\end{tabular} & $\begin{array}{l}1.00 \\
1.00 \\
1.00 \\
1.00\end{array}$ & $\begin{array}{l}0.98 \\
1.03 \\
1.04 \\
1.02 \\
0.02\end{array}$ & $\begin{array}{r}0.96 \\
1.00 \\
0.99 \\
0.98 \\
-0.02\end{array}$ & $\begin{array}{l}0.99 \\
1.00 \\
1.04 \\
1.01 \\
0.01\end{array}$ & $\begin{array}{l}0.98 \\
1.00 \\
0.98 \\
0.99 \\
-0.01\end{array}$ & $\begin{array}{l}1.03 \\
1.07 \\
1.05 \\
1.05 \\
0.05\end{array}$ & $\begin{array}{c}0.95 \\
1.05 \\
1.00 \\
1.00 \\
0\end{array}$ & $\begin{array}{c}1.00 \\
1.00 \\
1.00 \\
1.00 \\
0\end{array}$ & $\begin{array}{c}1.00 \\
1.00 \\
1.00 \\
0\end{array}$ \\
\hline \multirow{2}{*}{ Laparotomie } & $\mathrm{K}$-index & \begin{tabular}{l}
\multicolumn{1}{c}{1} \\
\multicolumn{2}{c}{3} \\
3 \\
Durchschn. \\
Differenz
\end{tabular} & $\begin{array}{l}45.2 \\
43.5 \\
40.7 \\
43.3\end{array}$ & $\begin{array}{l}38.3 \\
32.5 \\
50.0 \\
41.7 \\
-1.8\end{array}$ & $\begin{array}{l}27.0 \\
27.2 \\
41.2 \\
31.7 \\
-8.7\end{array}$ & $\begin{array}{r}33.3 \\
34.1 \\
45.7 \\
37.8 \\
0.3\end{array}$ & $\begin{array}{l}33.3 \\
31.0 \\
43.8 \\
36.1 \\
-4.9\end{array}$ & $\begin{array}{r}44.5 \\
34.1 \\
56.3 \\
43.7 \\
5.5\end{array}$ & $\begin{array}{r}49.5 \\
43.7 \\
50.8 \\
47.8 \\
4.8\end{array}$ & $\begin{array}{r}35.9 \\
64.2 \\
46.3 \\
47.8 \\
8.1\end{array}$ & $\begin{array}{l}44.6 \\
48.8 \\
40.4 \\
43.7 \\
-4.3\end{array}$ \\
\hline & P-zahl & \begin{tabular}{l}
\multicolumn{1}{c}{1} \\
\multicolumn{1}{c}{3} \\
3 \\
Durchschn. \\
Differenz
\end{tabular} & $\begin{array}{l}1.00 \\
1.00 \\
1.00 \\
1.00\end{array}$ & $\begin{array}{r}1.02 \\
0.97 \\
1.01 \\
1.00 \\
-0.02\end{array}$ & $\begin{array}{l}1.05 \\
1.05 \\
1.01 \\
1.04 \\
0.06\end{array}$ & $\begin{array}{r}1.02 \\
1.01 \\
0.98 \\
1.00 \\
-0.01\end{array}$ & $\begin{array}{l}1.06 \\
1.01 \\
1.00 \\
1.02 \\
0.03\end{array}$ & $\begin{array}{r}1.03 \\
0.98 \\
0.99 \\
1.00 \\
-0.05\end{array}$ & $\begin{array}{l}1.03 \\
0.98 \\
1.01 \\
1.01 \\
0.01\end{array}$ & $\begin{array}{l}1.03 \\
1.01 \\
1.02 \\
1.02 \\
0.02\end{array}$ & $\begin{array}{l}0.98 \\
1.04 \\
1.00 \\
1.01 \\
0.01\end{array}$ \\
\hline \multirow{2}{*}{$\begin{array}{l}\text { Milzexstirpa- } \\
\text { tion }\end{array}$} & $\mathrm{K}$-index & \begin{tabular}{l}
\multicolumn{1}{c}{1} \\
2 \\
Durchschn. \\
Differenz
\end{tabular} & $\begin{array}{l}53.2 \\
42.3 \\
48.4\end{array}$ & $\begin{array}{l}68.8 \\
68.2 \\
68.6 \\
21.8\end{array}$ & $\begin{array}{l}56.6 \\
41.3 \\
46.9 \\
10.1\end{array}$ & $\begin{array}{l}53.3 \\
63.8 \\
57.5 \\
14.6\end{array}$ & $\begin{array}{l}46.5 \\
38.7 \\
40.6 \\
-0.6\end{array}$ & $\begin{array}{r}41.7 \\
23.0 \\
34.0 \\
-14.8\end{array}$ & $\begin{array}{l}46.7 \\
53.9 \\
47.0 \\
-5.9\end{array}$ & $\begin{array}{l}45.8 \\
42.7 \\
44.4 \\
-8.5\end{array}$ & $\begin{array}{r}42.8 \\
56.7 \\
51.2 \\
2.4\end{array}$ \\
\hline & P-zahl & \begin{tabular}{l}
\multicolumn{1}{c}{1} \\
2 \\
Durchschn. \\
Differenz
\end{tabular} & $\begin{array}{l}1.00 \\
1.00 \\
1.00\end{array}$ & $\begin{array}{c}1.06 \\
0.93 \\
1.00 \\
0\end{array}$ & $\begin{array}{r}0.98 \\
1.05 \\
1.02 \\
-0.02\end{array}$ & $\begin{array}{c}0.98 \\
0.90 \\
0.94 \\
-0.06\end{array}$ & $\begin{array}{r}0.95 \\
0.99 \\
0.97 \\
-0.05\end{array}$ & $\begin{array}{l}1.00 \\
1.03 \\
1.01 \\
0.01\end{array}$ & $\begin{array}{l}1.06 \\
1.00 \\
1.03 \\
0.02\end{array}$ & $\begin{array}{r}0.98 \\
0.95 \\
0.97 \\
-0.05\end{array}$ & $\begin{array}{r}1.01 \\
1.00 \\
1.00 \\
-0.01\end{array}$ \\
\hline
\end{tabular}

Milzexstirpation reseziert, so sind eine Vermehrung des Kongorotindex und eine Verminderung der Prothrombinzahl auffälliger als bei 2 Leberlappenresektionen des gesunden Kaninchens.

Aus obigen Ergebnissen wird klar, dass die Funktion des RES gleich nach der Milzexstirpation herabgesetzt, dann kompensatorisch gesteigert wird und ungefähr nach 4 Wochen zum früheren Zustand zurückkehrt.

2. Einfluss der Milzpräparate

Aus Tabelle III sieht man, dass die Injektion des Extrakts einer ganzen Milz, der zentrifugierten Flüssigkeit der zergequetschten Kaninchenmilz mit $5 \mathrm{ccm}$ physiologischer Kochsalzlösung, unter die Haut des gesunden Kaninchens, 3 Stunden nach der Injektion eine Verminderung des Kongorotindex verursacht, wobei die Prothrombinzahl fast unverändert bleibt, während eine Verminderung des Kongorotindex bei subkutaner Injektion eines Fünftels des obigen Milzextrakts nicht so deutlich ist. 
TABELLE III

Einfluss der Milzpräparate

\begin{tabular}{|c|c|c|c|c|c|c|c|}
\hline & \multirow{2}{*}{$\begin{array}{l}\text { Dose } \\
\text { pro } \mathrm{kg}\end{array}$} & & \multirow{2}{*}{$\begin{array}{l}\text { Nr. des } \\
\text { Kaninchens }\end{array}$} & \multirow{2}{*}{$\begin{array}{l}\text { Vor } \\
\text { Inj. }\end{array}$} & \multicolumn{3}{|c|}{ Zeit nach Injektion (St.) } \\
\hline & & & & & 1 & 3 & 5 \\
\hline \multirow{4}{*}{ Milzextrakt } & \multirow{2}{*}{$\begin{array}{c}\text { ganze } \\
\text { Menge }\end{array}$} & $\mathrm{K}$-index & \begin{tabular}{l}
\multicolumn{1}{c}{1} \\
2 \\
Durchschn. \\
Differenz
\end{tabular} & $\begin{array}{l}52.0 \\
51.7 \\
51.9\end{array}$ & $\begin{array}{r}68.0 \\
59.5 \\
63.8 \\
7.4\end{array}$ & $\begin{array}{r}42.6 \\
46.2 \\
44.5 \\
-18.4\end{array}$ & $\begin{array}{r}43.3 \\
60.8 \\
52.3 \\
-16.1\end{array}$ \\
\hline & & P-zahl & \begin{tabular}{l}
\multicolumn{1}{c}{1} \\
\multicolumn{2}{c}{} \\
Durchschn. \\
Differenz
\end{tabular} & $\begin{array}{l}1.00 \\
1.00 \\
1.00\end{array}$ & $\begin{array}{r}1.03 \\
0.94 \\
0.99 \\
-0.01\end{array}$ & $\begin{array}{r}1.00 \\
0.97 \\
0.99 \\
-0.03\end{array}$ & $\begin{array}{r}0.97 \\
1.03 \\
1.00 \\
-0.04\end{array}$ \\
\hline & \multirow{2}{*}{$\begin{array}{c}1 / 5 \\
\text { Menge }\end{array}$} & $\mathrm{K}$-index & $\begin{array}{l}1 \\
1 \\
\text { Durchschn. } \\
\text { Differenz }\end{array}$ & $\begin{array}{l}46.0 \\
45.1 \\
45.7\end{array}$ & $\begin{array}{r}53.0 \\
50.6 \\
51.9 \\
1.7\end{array}$ & $\begin{array}{l}60.2 \\
52.2 \\
56.6 \\
-0.1\end{array}$ & $\begin{array}{l}53.5 \\
54.0 \\
53.7 \\
-8.4\end{array}$ \\
\hline & & P-zahl & \begin{tabular}{l}
\multicolumn{1}{c}{1} \\
2 \\
Durchschn. \\
Differenz
\end{tabular} & $\begin{array}{l}1.00 \\
1.00 \\
1.00\end{array}$ & $\begin{array}{c}0.99 \\
1.01 \\
1.00 \\
0\end{array}$ & $\begin{array}{r}0.99 \\
0.98 \\
0.99 \\
-0.03\end{array}$ & $\begin{array}{r}1.03 \\
1.02 \\
1.03 \\
-0.01\end{array}$ \\
\hline \multirow{2}{*}{ Milzsin } & \multirow{2}{*}{$\begin{array}{l}1 \mathrm{ccm} \\
\text { s.c. }\end{array}$} & K-index & \begin{tabular}{l}
\multicolumn{1}{c}{1} \\
\multicolumn{2}{c}{} \\
Durchschn. \\
Differenz
\end{tabular} & $\begin{array}{l}66.8 \\
54.6 \\
60.7\end{array}$ & $\begin{array}{r}55.4 \\
53.1 \\
54.3 \\
-18.1\end{array}$ & $\begin{array}{r}69.4 \\
60.0 \\
65.0 \\
-14.8\end{array}$ & $\begin{array}{l}80.0 \\
73.3 \\
76.8 \\
-1.4\end{array}$ \\
\hline & & P-zahl & \begin{tabular}{l}
\multicolumn{1}{c}{1} \\
2 \\
Durchschn. \\
Differenz
\end{tabular} & $\begin{array}{l}1.00 \\
1.00 \\
1.00\end{array}$ & $\begin{array}{l}1.00 \\
0.99 \\
1.00 \\
0.01\end{array}$ & $\begin{array}{l}1.00 \\
0.98 \\
0.99 \\
0.02\end{array}$ & $\begin{array}{r}0.95 \\
0.96 \\
0.96 \\
-0.02\end{array}$ \\
\hline \multirow{2}{*}{ Retio A } & \multirow{2}{*}{$\begin{array}{l}1 \mathrm{EH} \\
\text { s.c. }\end{array}$} & $\mathrm{K}$-index & \begin{tabular}{l}
\multicolumn{1}{c}{} \\
2 \\
Durchschn. \\
Differenz
\end{tabular} & $\begin{array}{l}45.9 \\
42.7 \\
44.3\end{array}$ & $\begin{array}{r}35.5 \\
41.6 \\
38.2 \\
-9.3\end{array}$ & $\begin{array}{l}51.1 \\
43.7 \\
47.4 \\
-1.1\end{array}$ & $\begin{array}{l}46.6 \\
50.0 \\
47.9 \\
-0.1\end{array}$ \\
\hline & & P-zahl & $\begin{array}{l}1 \\
1 \\
2 \\
\text { Durchschn. } \\
\text { Differenz }\end{array}$ & $\begin{array}{l}1.00 \\
1.00 \\
1.00\end{array}$ & $\begin{array}{r}0.99 \\
0.99 \\
0.99 \\
-0.02\end{array}$ & $\begin{array}{r}1.02 \\
0.99 \\
1.00 \\
-0.03\end{array}$ & $\begin{array}{r}0.98 \\
0.97 \\
0.98 \\
-0.05\end{array}$ \\
\hline
\end{tabular}

Wenn man Milzsin (Nippon Shinyaku), das Milzhormon, in Menge von $1 \mathrm{ccm}$ pro $\mathrm{kg}$, oder Retio A (Takenaka Seiyaku), das Autohormon der reticuloendothelialen Zellen, in Menge von 1 Einheit unter die Haut des gesunden Kaninchens injiziert, so vermindert sich der Kongorotindex vorübergehend, die Prothrombinzahl bleibt aber fast unverändert.

Wie man aus Tabelle IV ersieht, sind eine Verminderung des Kongorotindex und eine Vermehrung der Prothrombinzahl bei subkutaner Injektion des Extrakts einer ganzen Milz 5 Stunden nach der Milzexstirpation sehr merklich, und sogar der Kongorotindex vermindert sich 
TABELLE IV

Einfluss der Milzpräparate nach der Milzexstirpation

\begin{tabular}{|c|c|c|c|c|c|c|c|c|}
\hline & \multirow{2}{*}{$\begin{array}{c}\text { Dose } \\
\text { pro } \mathrm{kg}\end{array}$} & & \multirow{2}{*}{$\begin{array}{l}\text { Nr. des } \\
\text { Kaninchens }\end{array}$} & \multirow{2}{*}{$\begin{array}{c}\text { Vor } \\
\text { Milz- } \\
\text { exstir- } \\
\text { pation }\end{array}$} & \multirow{2}{*}{$\begin{array}{l}5 \text { St. } \\
\text { nach } \\
\text { Milz- } \\
\text { exstir- } \\
\text { pation }\end{array}$} & \multicolumn{3}{|c|}{$\begin{array}{c}\text { Zeit nach Injektion } \\
\text { (St.) }\end{array}$} \\
\hline & & & & & & 1 & 3 & 5 \\
\hline \multirow{2}{*}{$\begin{array}{l}\text { Milzexstir- } \\
\text { pation }\end{array}$} & & $\mathrm{K}$-index & \begin{tabular}{l}
$\quad 1$ \\
\multicolumn{1}{c}{} \\
\multicolumn{1}{c}{ Durchschn. } \\
Differenz
\end{tabular} & $\begin{array}{l}45.9 \\
42.1 \\
44.0\end{array}$ & $\begin{array}{l}53.8 \\
46.5 \\
49.5\end{array}$ & $\begin{array}{r}50.0 \\
55.8 \\
53.0 \\
3.5\end{array}$ & $\begin{array}{r}52.4 \\
52.3 \\
52.5 \\
2.8\end{array}$ & $\begin{array}{r}61.4 \\
55.0 \\
57.7 \\
8.2\end{array}$ \\
\hline & & P-zahl & \begin{tabular}{l}
1 \\
\multicolumn{1}{c}{1} \\
Durchschn. \\
Differenz
\end{tabular} & $\begin{array}{l}1.00 \\
1.00 \\
1.00\end{array}$ & $\begin{array}{c}1.04 \\
1.02 \\
1.03 \\
(1.00)\end{array}$ & $\begin{array}{r}1.00 \\
0.98 \\
0.99 \\
-0.01\end{array}$ & $\begin{array}{r}0.98 \\
0.98 \\
0.98 \\
-0.02\end{array}$ & $\begin{array}{r}0.99 \\
0.99 \\
0.99 \\
-0.01\end{array}$ \\
\hline \multirow{2}{*}{$\begin{array}{l}\text { Milzextrakt } \\
\text { nach } \\
\text { Milzexstir- } \\
\text { pation }\end{array}$} & \multirow{2}{*}{$\begin{array}{l}\text { ganze } \\
\text { Menge }\end{array}$} & K-index & \begin{tabular}{l}
\multicolumn{1}{c}{1} \\
\multicolumn{2}{c}{} \\
Durchschn. \\
Differenz
\end{tabular} & $\begin{array}{l}72.1 \\
57.7 \\
63.1\end{array}$ & $\begin{array}{l}85.1 \\
68.6 \\
73.3\end{array}$ & $\begin{array}{r}59.5 \\
65.0 \\
62.8 \\
-14.0\end{array}$ & $\begin{array}{l}73.3 \\
70.6 \\
71.7 \\
-4.4\end{array}$ & $\begin{array}{r}60.8 \\
73.0 \\
68.3 \\
-13.2\end{array}$ \\
\hline & & P-zahl & \begin{tabular}{l}
\multicolumn{1}{c}{1} \\
\multicolumn{2}{c}{} \\
Durchschn. \\
Differenz
\end{tabular} & $\begin{array}{l}1.00 \\
1.00 \\
1.00\end{array}$ & $\begin{array}{c}1.02 \\
1.01 \\
1.01 \\
(1.00)\end{array}$ & $\begin{array}{l}1.18 \\
1.01 \\
1.09 \\
0.10\end{array}$ & $\begin{array}{l}1.21 \\
1.00 \\
1.10 \\
0.12\end{array}$ & $\begin{array}{l}1.22 \\
1.03 \\
1.12 \\
0.13\end{array}$ \\
\hline \multirow{2}{*}{$\begin{array}{l}\text { Milzsin } \\
\text { nach } \\
\text { Milzexstir- } \\
\text { pation }\end{array}$} & \multirow{2}{*}{$\begin{array}{l}1 \mathrm{ccm} \\
\text { s.c. }\end{array}$} & $\mathrm{K}$-index & $\begin{array}{c}1 \\
2 \\
3 \\
\text { Durchschn. } \\
\text { Differenz }\end{array}$ & $\begin{array}{l}52.2 \\
51.4 \\
41.1 \\
48.2\end{array}$ & $\begin{array}{l}59.8 \\
65.8 \\
73.2 \\
67.2\end{array}$ & $\begin{array}{l}60.8 \\
71.2 \\
74.8 \\
69.8 \\
-0.9\end{array}$ & $\begin{array}{l}59.1 \\
66.7 \\
66.7 \\
64.4 \\
-5.6\end{array}$ & $\begin{array}{l}65.0 \\
75.0 \\
66.7 \\
68.8 \\
-6.6 \\
\end{array}$ \\
\hline & & P-zahl & 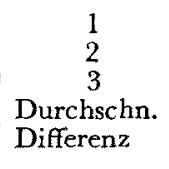 & $\begin{array}{l}1.00 \\
1.00 \\
1.00 \\
1.00\end{array}$ & $\begin{array}{c}0.98 \\
0.98 \\
1.01 \\
0.99 \\
(1.00)\end{array}$ & $\begin{array}{l}1.01 \\
1.03 \\
0.97 \\
1.00 \\
0.01\end{array}$ & $\begin{array}{l}1.02 \\
1.07 \\
1.01 \\
1.03 \\
0.05\end{array}$ & $\begin{array}{l}1.08 \\
1.06 \\
1.06 \\
1.07 \\
0.08\end{array}$ \\
\hline \multirow{2}{*}{$\begin{array}{l}\text { Glukose } \\
\text { nach } \\
\text { Milzexstir- } \\
\text { pation }\end{array}$} & \multirow{2}{*}{$\begin{array}{l}25 \% 10 \mathrm{ccm} \\
\text { i.v. }\end{array}$} & K-index & \begin{tabular}{l}
\multicolumn{1}{c}{1} \\
\multicolumn{1}{c}{2} \\
Durchschn. \\
Differenz
\end{tabular} & $\begin{array}{l}63.8 \\
56.6 \\
60.3\end{array}$ & $\begin{array}{l}88.8 \\
89.6 \\
89.3\end{array}$ & $\begin{array}{r}64.3 \\
73.3 \\
67.3 \\
-25.5 \\
\end{array}$ & $\begin{array}{r}80.3 \\
78.4 \\
79.6 \\
-12.5 \\
\end{array}$ & $\begin{array}{r}83.3 \\
65.2 \\
73.1 \\
-24.4\end{array}$ \\
\hline & & P-zahl & \begin{tabular}{l}
$\quad 1$ \\
\multicolumn{1}{c}{${ }^{2}$} \\
Durchschn. \\
Differenz
\end{tabular} & $\begin{array}{l}1.00 \\
1.00 \\
1.00\end{array}$ & $\begin{array}{c}0.99 \\
0.99 \\
0.99 \\
(1.00)\end{array}$ & $\begin{array}{r}0.98 \\
0.97 \\
0.98 \\
-0.01\end{array}$ & $\begin{array}{l}1.00 \\
1.00 \\
1.00 \\
0.02\end{array}$ & $\begin{array}{l}1.04 \\
0.98 \\
1.01 \\
0.02\end{array}$ \\
\hline \multirow{2}{*}{$\begin{array}{l}\text { Hypon } \\
\text { nacn } \\
\text { Milzexstir- } \\
\text { pation }\end{array}$} & \multirow{2}{*}{$\begin{array}{l}50 \% 1 \mathrm{ccm} \\
\text { i.v. }\end{array}$} & $\mathrm{K}$-index & \begin{tabular}{l}
\multicolumn{1}{c}{1} \\
\multicolumn{2}{|c}{} \\
Durchschn. \\
Differenz
\end{tabular} & $\begin{array}{l}65.2 \\
51.2 \\
56.9\end{array}$ & $\begin{array}{l}88.8 \\
71.7 \\
79.4\end{array}$ & $\begin{array}{r}56.8 \\
69.5 \\
63.2 \\
-19.7\end{array}$ & $\begin{array}{l}72.8 \\
78.8 \\
75.8 \\
-6.4\end{array}$ & $\begin{array}{r}75.0 \\
82.3 \\
77.3 \\
-10.3\end{array}$ \\
\hline & & P-zahl & \begin{tabular}{l}
\multicolumn{1}{c}{1} \\
\multicolumn{1}{c}{2} \\
Durchschn. \\
Differenz
\end{tabular} & $\begin{array}{l}1.00 \\
1.00 \\
1.00\end{array}$ & $\begin{array}{l}1.03 \\
0.98 \\
1.00\end{array}$ & $\begin{array}{l}0.97 \\
1.07 \\
1.02 \\
0.03\end{array}$ & $\begin{array}{l}0.99 \\
1.01 \\
1.00 \\
0.02\end{array}$ & $\begin{array}{r}0.95 \\
1.01 \\
0.98 \\
-0.01\end{array}$ \\
\hline
\end{tabular}


leichtgradig, die Prothrombinzahl aber vermehrt sich nach Milzsininjektion beim entmilzten Kaninchen. Aber die Injektion von $25 \%$ Glukose in Menge von $10 \mathrm{ccm}$ pro $\mathrm{kg}$ oder von $50 \%$ Hypon (Natriumthiosulfat) in Menge von $1 \mathrm{ccm}$ pro $\mathrm{kg}$ in die Vene des entmilzten Kaninchens verursacht eine Verminderung des Kongorotindex, während die Prothrombinzahl fast unverändert bleibt.

Aus diesen Versuchsergebnissen ist zu schliessen, dass diese Milzpräparate einen günstigen Einfluss auf die Funktion des RES sowohl beim gesunden als auch beim entmilzten Kaninchen ausüben.

\section{Diskussion}

Nach Aschoff ${ }^{11}$ gehören die Reticulmzellen der Milzpulpa und die Sinusendothelien der Milz zum RES im engeren Sinne und die Splenozyten zum RES im weiteren Sinne; deshalb wird allgemein anerkannt, dass die Milz ein wichtiges Organ des RES ist. Mehrere Autoren nehmen an, dass die Milzexstirpation die Funktion des RES herabsetzt (Adler, ${ }^{5}$ ) Leites, ${ }^{61}$ Yuki ${ }^{7}$, Tokuno, ${ }^{81}$ Kinoshita, ${ }^{91}$ Goebel und Miller $\left.{ }^{10)}\right)$. Aber Noma $^{11)}$ und Tomoda ${ }^{12}$ ) betonen, dass diese Herabsetzung der Funktion des RES nach der Milzexstirpation durch sonstige reticuloendotheliale Zellen wie Kupffersche Sternzellen in der Leber kompensiert werden kann. Weiter weisen Goebel ${ }^{10)}$ und Rindone ${ }^{13}$ histologisch nach, dass die Sternzellen nach der Milzexstirpation hypertrophieren und wuchern.

Aus unseren Versuchsergebnissen ist es klar geworden, dass die Funktion des RES sofort nach der Milzexstirpation herabgesetzt, dann kompensatorisch gesteigert wird und ungefähr nach 4 Wochen zum früheren Zustand zurückkehrt. Dass die Leberzellenfunktion mehr oder weniger zu einer Verminderung neigt, nicht nur nach der Milzexstirpation, sondern auch nach 2 Leberlappenresektionen des entmilzten Kaninchens, lässt vermuten, dass die Milz eine kompensatorische Funktion für die Leber hat.

Schon in der II. Mitteilung ${ }^{3)}$ dieser Arbeit fanden wir, dass die intravenöse Injektion von Glukose oder Natriumthiosulfat die Funktion des RES steigert. Aber Tokuno ${ }^{8)}$ bemerkte, dass das Milzextrakt die Funktion des RES erhöht, und weiter betonte Torrioli, ${ }^{14)}$ dass die reticuloendothelialen Zellen durch die intravenöse Injektion des Milzextrakts wuchern.

Nach unseren diesbezüglichen Ergebnissen wird festgestellt, dass das Milzextrakt, Milzsin und Retio A zwar nur die Funktion des RES des gesunden Kaninchens steigern kann, aber das Milzextrakt und Milzsin sowohl die Funktion des RES als auch die Leberzellenfunktion des entmilzten Kaninchens erhöhen, während Glukose und Hypon nur die Funk- 
tion des RES des gesunden sowie des entmilzten Kaninchens fördern kann.

Aus obenerwähnten Versuchsergebnissen ist zu vermuten, dass die Milz an der Funktion des RES beteiligt ist, und zwar nicht nur als Teilorgan des RES, sondern auch als inkretorisches Organ des stimulierenden Hormons des RES.

\section{ZUSAMMENFASSUNG}

Fassen wir obige Versuchsergebnisse zusammen, so kommen wir zu folgendem Schluss :

1. Nach der Milzexstirpation vermehrt sich der Kongorotindex bis etwa zum 7. Tage, dann vermindert er sich und kehrt ungefähr nach 4 Wochen zum früheren Zustand wieder zurück, während eine Verminderung der Prothrombinzahl nicht so auffallend ist.

2. Nach 2 Leberlappenresektionen des entmilzten Kaninchens sind eine Verminderung des Kongorotindex und eine Verminderung der Prothrombinzahl auffälliger als diejenigen des gesunden Kaninchens.

3. Bei Injektion von Milzextrakt, Milzsin und Retio A vermindert sich der Kongorotindex des gesunden Kaninchens, während die Prothrombinzahl fast unverändert bleibt. Aber eine Verminderung des Kongorotindex und eine Vermehrung der Prothrombinzahl des entmilzten Kaninchens sind sehr merklich bei Injektion von Milzextrakt und Milzsin. Weiter bei Injektion von Glukose und Hypon in die Vene des entmilzten Kaninchens vermindert sich der Kongorotindex, die Prothrombinzahl bleibt aber fast unverändert.

4. Daraus ist zu vermuten, dass die Funktion des RES sich sofort nach der Milzexstirpation herabsetzt, dann kompensatorisch sich steigert, um schliesslich den früheren Zusand wiederherzustellen, und dass die Milz nicht nur einen stimulierenden Faktor für die Funktion des RES enthält, sondern auch ein stimulierendes Hormon sezerniert.

\section{Literatur}

1) Aschoff, Erg. inn. Med., 1924, 26, 1.

2) Akazaki, Transact. Jap. Path. Soc., 1952, 41, 1.

3) Yamagata et al., J. Jap. Soc. Int. Med. (Jap.), 1952, 41, 341.

4) Yamagata et al., Ebenda, 1952, 41, 338.

5) Adler \& Reimann, Zschr. exper. Med., 1925, 47, 617.

6) Leites \& Riabow, Ebenda, 1927, 58, 314.

7) Yuki, Mitt. Jap. Ges. Gynäk., 1928, 23, 1413.

8) Tokuno, J. Jap. Surg. Soc. (Jap.), 1931, 32, 104.

9) Kinoshita, Mitt. Med. Akad. Kioto (Jap.), 1933, 8, 1040.

10) Goebel \& Miller, Compt. rend. soc. biol., 1935, 115, 775.

11) Noma, Mitt. Okayama Med. Ges. (Jap.), 1925, Nr. 428, 929. 
12) Tomoda, Ketsueki Togikai Hokoku (Jap.), 1949, 2. Aufl., 80.

13) Rindone, Osp. maggiore, 1940, 28, 398.

14) Torrioli, Policlinico sez. med., 1939, 46, 337. 